\title{
Calculation of the Rovibrational Partition Function Using Classical Methods with Quantum Corrections
}

\author{
Frederico V. Prudente, Antonio Riganelli, and António J. C. Varandas* \\ Departamento de Química, Universidade de Coimbra, P-3049 Coimbra Codex, Portugal
}

Received: December 6, 2000; In Final Form: February 28, 2001

\begin{abstract}
The rovibrational partition functions of diatomic molecules are calculated using a classical framework plus quantum, semiclassical, and semiempirical corrections. The most popular methods to calculate such corrections are briefly reviewed and applied to the benchmark $\mathrm{H}_{2}$ molecule. A novel hybrid scheme is proposed and applied to $\mathrm{H}_{2}, \mathrm{HCl}$, and ArO. Each method is analyzed with a view to find an economical way to calculate such corrections for polyatomic systems.
\end{abstract}

\section{Introduction}

Accurate values of the rovibrational partition function $\left(q_{\mathrm{vr}}\right)$ are frequently needed in chemistry and physics. They are required to calculate the equilibrium properties of molecular systems, ${ }^{1}$ and the rates of chemical reactions using transition state theory. ${ }^{2,3}$ In principle, $q_{\mathrm{vr}}$ can be evaluated exactly in quantum statistical mechanics by carrying out the following explicit summation of Boltzmann factors

$$
q_{\mathrm{vr}}^{\mathrm{QM}}(T)=\sum_{i} g_{i} \exp \left(-\beta \epsilon_{i}\right)
$$

where $\beta=1 /\left(k_{\mathrm{B}} T\right), k_{\mathrm{B}}$ is the Boltzmann constant, $T$ is the temperature, $\epsilon_{\mathrm{i}}$ is the rovibrational energy associated with state $i$, and $g_{i}$ is the corresponding degeneracy factor. However, the calculation of highly excited states with spectroscopic accuracy is currently feasible only for systems with a few degrees of freedom, ${ }^{4-6}$ which limits the evaluation of the rovibrational partition function using eq 1 to small molecules and low rotational states. ${ }^{7-10}$

In turn, $q_{\mathrm{vr}}$ assumes in classical statistical mechanics the form ${ }^{1,11}$

$$
q_{\mathrm{vr}}^{\mathrm{CM}}(T)=\frac{1}{h^{n}} \iint_{\mathrm{B}} \exp \left\{-\beta H^{\mathrm{CM}}(\mathbf{q}, \mathbf{p})\right\} \mathrm{d} \mathbf{q} \mathrm{d} \mathbf{p}
$$

with $H^{\mathrm{CM}}(\mathbf{q}, \mathbf{p})$ being the classical Hamiltonian, $h$ the Planck constant, $n$ the number of degrees of freedom, $\mathbf{q}$ the generalized coordinate vector, and $\mathbf{p}$ its conjugate momenta. In turn, the subscript $B$ implies that the hypervolume of integration is restricted to phase space regions corresponding to a bound state situation, i.e., $0 \leq H^{\mathrm{CM}}(\mathbf{q}, \mathbf{p}) \leq D_{\mathrm{e}}$, where $D_{\mathrm{e}}$ is the dissociation energy of the molecule ${ }^{12}$ (throughout this work we assume as reference energy the minimum of the potential energy surface). A major advantage of the classical approach is the appreciably smaller computational cost in comparison to the quantum one, which allows a treatment of molecular systems with a large number of degrees of freedom. Yet, it is well-known that $q_{\mathrm{vr}}^{\mathrm{CM}}$ overestimates $q_{\mathrm{vr}}^{\mathrm{QM}}$ at low temperatures, while converging to the latter at the high-temperature limit; ${ }^{1}$ for a recent discussion on the range of applicability of classical statistical mechanics to calculate the vibrational partition function for triatomic systems, see ref 13 .

For molecular simulations it is essential to have an economical recipe to introduce corrections into the partition functions evaluated within the classical framework. The idea for introducing such corrections comes from the early days of quantum mechanics. Such corrections can be based on "strictly" quantum, semiclassical and semiempirical formulations. A well established strictly quantum approach comes from the seminal work of Wigner ${ }^{14}$ and Kirkwood. ${ }^{15}$ They have shown that the quantum probability function (and hence the quantum partition function) in phase space can be obtained through an expansion in powers of $\hbar=h / 2 \pi$. The Wigner-Kirkwood (WK) expansion has been extensively employed in molecular simulations of liquids, ${ }^{16-19}$ and to calculate the partition functions of hindered rotors ${ }^{20}$ and diatomic molecules. ${ }^{21,22}$ Another procedure to correct $q_{\mathrm{vr}}^{\mathrm{CM}}$ from quantum mechanics is due to Green ${ }^{23}$ and Oppenheim and Ross $^{24}$ (GOR). Similar to the WK method, the GOR approach consists of writing the quantum Hamiltonian within the phase space formalism as an expansion in powers of $\hbar$, which is then used in eq 2; for applications of the GOR method in molecular simulations, see ref 25 .

Other approaches to correct the classical partition function come from path integral formulations, ${ }^{26,27}$ and are of semiclassical nature. One such a proposal due to Feynman and Hibbs ${ }^{27}$ uses the Feynman path integral formulation of quantum mechanics, and consists of approximating the integration of the energy functional over all paths by using an effective potential. Since their proposal, several forms of the effective potential have been suggested and applied to various physical problems, ${ }^{19,28-34}$ including the calculation of the molecular rovibrational partition function. ${ }^{35}$ The second proposal comes from Miller and co-workers ${ }^{36-39}$ and is based on an approximation of the Feynman path integrals by their classical counterpart which, are in turn approximated by using a "semiclassical" potential. Such an approach has been utilized in the context of molecular vibration-rotation dynamics. ${ }^{40}$

A simple semiempirical procedure to correct the classical partition function was suggested by Pitzer and Gwinn. ${ }^{41}$ In the Pitzer-Gwinn method, the quantum partition function is approximated as the classical partition function scaled by the ratio of the quantum and classical partition functions for a reference 
system in which the later assume analytical form. Usually the reference system is a generalized harmonic oscillator potential. This is a very useful approximation, with several variants of it having been tested for realistic potential energy surfaces. ${ }^{22,28,35,42-45}$

The main goal of the present work is to study the efficiency of the above methods and discuss their advantages and disadvantages in molecular partition function calculations. We also seek to establish a simple procedure in which the computational effort is relatively inexpensive and easy to generalize to multidimensional systems. This employs an hybrid effective potential which is built from effective potentials reported by previous authors. Our purpose follows a claim made in previous work $^{13,46}$ (hereafter referred to as papers I and II) to seek a general economical procedure for calculating accurate values of internal partition functions based on realistic potential energy surfaces.

For the evaluation of the phase space or configurational integrals which arise from the quantum, semiclassical, or semiempirical corrections discussed here we employ the method described in papers I and II. The method is based on a Monte Carlo technique proposed by Barker to calculate the density of states in transition state theory. ${ }^{47} \mathrm{We}$ have limited our study to diatomic systems since exact quantum partition functions can be easily computed to test the methodologies discussed in the present work. However, both the approaches to correct $q_{\mathrm{vr}}^{\mathrm{CM}}$ and the Monte Carlo technique are given for the general $n$-dimensional case.

As case studies, we consider $\mathrm{H}_{2}, \mathrm{HCl}$, and $\mathrm{ArO}$ in their ground electronic states. While the first two systems represent chemically stable species with different well depths, the latter is a weakly bound van der Waals molecule. For $\mathrm{H}_{2}$, we have tested numerically all the methods reported above. Such a systematic study will help us to figure out a novel scheme to introduce quantum corrections into the classical partition function. This new approach is then tested on the $\mathrm{HCl}$ and $\mathrm{ArO}$ molecules. For $\mathrm{H}_{2}$, the calculations are limited to the vibrational partition function to avoid the inclusion of nuclear spin degeneracy factors which arise when the rotational motion is considered. Moreover as pointed out by Taubmann et al., ${ }^{22}$ an exact calculation of the vibrational partition function for $\mathrm{H}_{2}$ is a rather challenging task due to the large anharmonicity of the associated potential energy curve. On the other hand, the breakdown of the classical picture is more serious for vibrational than rotational motions, since rotational quanta are much smaller than vibrational ones. However, for $\mathrm{HCl}$, and $\mathrm{ArO}$, we report calculations of the full rovibrational partition function.

The paper is organized as follows. In section 2, we summarize the technical details. This includes an outline of the Monte Carlo technique used in the classical calculations, a description of the potential energy curves, and the details referring to the calculation of the rovibrational energy levels. Section 3 reviews the various methods to include corrections in the classical vibrational partition function: the Wigner-Kirkwood (WK) expansion, Green-Oppenheim-Ross (GOR) expansion, linear approximation of the classical path (LCP) approach, quadratic FeynmanHibbs (QFH) approximation of Feynman path integral, and the Pitzer-Gwinn (PG) approximation. A novel hybrid LCP/QFH method is also reported in the present work is reported in section 4. Some conclusions are in section 5 .

\section{Technical Details}

2.1. Monte Carlo Procedure. For all calculations reported in sections 3 and 4 , it will be necessary to solve a multidimen- sional integral of the following general form:

$$
I=\int \ldots \int_{\mathrm{B}} f\left(x_{1}, \ldots, x_{k}\right) \mathrm{d} x_{1} \ldots \mathrm{d} x_{k}
$$

where $\left\{x_{i}\right\}$ are variables of the integrand function $f$, and B stands for an appropriate volume of integration in $(k-1)$-dimensions defined by

$$
B=\mathrm{F}\left(x_{1}, \ldots, x_{k}\right)
$$

where $B$ is a scalar and $\mathrm{F}$ a $k$-dimensional function. An example is the classical partition function, for which we have employed $^{13,46}$ an efficient Monte Carlo scheme to evaluate it for polyatomic systems. This method is an adaptation of Barker's algorithm, ${ }^{47}$ which is based on stratified (guided) sampling procedures. The basic idea is to choose a sampling domain which coincides, as much as possible, with the integration domain. Thus, the variables will not be sampled independently. Instead, one establishes a hierarchy into the sampling procedure such that the efficiency of the Monte Carlo method is maximized. As a result, the sampled points form a normalized but nonuniform distribution, requiring the use of weighting factors.

The algorithm may be summarized in the following steps: 1. Find $\tilde{\mathbf{x}}=\left(\tilde{x}_{1}, \ldots, \tilde{x}_{k}\right)$ which defines the minimum of $\mathrm{F}\left(x_{1}\right.$, $\left.\ldots, x_{k}\right)$.

2. Find the interval $\left(x_{1}^{\min }, x_{1}^{\max }\right)$ for variable $x_{1}$ with all other variables fixed at the values obtained in step 1 , according to

$$
\mathrm{F}\left(x_{1}^{\min }, \tilde{x}_{2}, \ldots, \tilde{x}_{k}\right)=\mathrm{F}\left(x_{1}^{\max }, \tilde{x}_{2}, \ldots, \tilde{x}_{k}\right)=B
$$

3. Sample randomly $x_{1}$ within this range to obtain $x_{1}^{S}$, according to

$$
x_{1}^{\mathrm{S}}=x_{1}^{\min }+\left(x_{1}^{\max }-x_{1}^{\min }\right) \xi
$$

where $\xi$ is a random number between 0 and 1 .

4. Starting with $i=2$, find new values for the remaining ( $k$ $-i+1)$ variables which define the minimum $\mathrm{F}\left(x_{1}^{\mathrm{S}}, \ldots, x_{i-1}^{\mathrm{S}}, x_{i}\right.$, $\left.\ldots, x_{k}\right)$

5. Find the integration domain $\left(x_{i}^{\min }, x_{i}^{\max }\right)$ for $x_{i}$ with the first $(i-1)$ variables fixed at the sampled values and the other $(k-i)$ variables at the values obtained in step 4 , according to

$$
\begin{gathered}
\mathrm{F}\left(x_{1}^{\mathrm{S}}, \ldots, x_{i-1}^{\mathrm{S}}, x_{i}^{\min }, \tilde{x}_{i+1}, \ldots, \tilde{x}_{k}\right) \\
\mathrm{F}\left(x_{1}^{\mathrm{S}}, \ldots, x_{i-1}^{\mathrm{S}}, x_{i}^{\max }, \tilde{x}_{i+1}, \ldots, \tilde{x}_{k}\right)=B
\end{gathered}
$$

6. Sample randomly $x_{i}$ inside this range to obtain $x_{i}^{\mathrm{S}}$ as in step 3.

7. Repeat steps $4-6$ for $i=3, \ldots, K$ until the value $\left(x_{k}^{\mathrm{S}}\right)$ of the last variable is sampled. This will result in the selection of a sampled point within the boundary surface $B$ defined by eq 4. Note that the range of the $i$ th variable is conditioned with respect to all values of the variables previously selected.

8. Calculate the weight factor for each sampled point $g, \mathbf{x}_{g}^{\mathrm{S}}$ $=\left(x_{1}^{S}, \ldots, x_{k}^{S}\right)$, according to

$$
w_{g}=\prod_{i=1}^{k}\left(x_{i}^{\max }-x_{i}^{\min }\right)
$$

which represents the hypervolume associated to $\mathbf{x}_{g}^{S}$. 
TABLE 1: Parameters for the Morse Potential of $\mathrm{H}_{2}:{ }^{65}$ All Quantities in au

$$
\begin{aligned}
& D_{\mathrm{e}}=0.1744 \\
& \omega=1.02764 \\
& r_{\mathrm{e}}=1.40201 \\
& \mu=918.6446
\end{aligned}
$$

9. Repeat steps 3-8 $M$ times to calculate the integral in eq 3 as

$$
I \approx I^{M}=\frac{1}{M} \sum_{g=1}^{M} w_{g} f_{g}
$$

where $f_{g}=f\left(\mathbf{x}_{g}^{\mathrm{S}}\right)$ is the function to be integrated and $M$ is the total number of sampled points. The standard deviation associated with eq 9 is ${ }^{47}$

$$
\sigma^{2}=\frac{1}{M(M-1)} \sum_{g=1}^{M}\left(w_{g} f_{g}-I^{M}\right)^{2}
$$

2.2. Potential Energy Curves. We summarize in this section the potential energy curves used for $\mathrm{H}_{2}, \mathrm{HCl}$, and $\mathrm{ArO}$. Although more sophisticated models ${ }^{48,49}$ are available for $\mathrm{H}_{2}$ and $\mathrm{HCl}$, we have adopted curves which have been extensively used by others in calculations of the partition function.

The $\mathrm{H}_{2}$ molecule can be a benchmark system for such an application due to its strong anharmonicity, as discussed by Taubmann et al. ${ }^{22}$ It will be described by a Morse curve ${ }^{50}$ defined by

$$
V(r)=D_{\mathrm{e}}\left\{1-\exp \left[-\omega\left(r-r_{\mathrm{e}}\right)\right]\right\}^{2}
$$

where $D_{e}$ is the equilibrium dissociation energy, $\omega$ a parameter, and $r_{\mathrm{e}}$ the equilibrium interatomic distance; Table 1 gathers the numerical values of the involved parameters. For this model, the vibrational spectrum is given analytically as ${ }^{51}$

$$
\begin{aligned}
E_{v}=D_{\mathrm{e}}\left[\left(v+\frac{1}{2}\right)\right] \sqrt{\frac{2 \omega^{2} \hbar^{2}}{D_{\mathrm{e}} \mu}}-\left[\left(v+\frac{1}{2}\right)\right]^{1 / 2} \frac{\omega^{2} \hbar^{2}}{2 \mu} & \\
v & =0,1, \ldots
\end{aligned}
$$

under the condition

$$
v<\frac{\sqrt{2 \mu D_{\mathrm{e}}}}{\hbar \omega}-\frac{1}{2}
$$

This leads to a total of 17 vibrational bound states for $\mathrm{H}_{2}$

For $\mathrm{HCl}$, we employ the empirical Hulbert-Hirschfelder curve $^{52}$ defined by

$$
V(r)=D_{\mathrm{e}}\left[\left(1-\mathrm{e}^{-y}\right)^{2}+c y^{3} \mathrm{e}^{-2 y}(1+b y)\right]
$$

where $y$ is the reduced internuclear distance

$$
y(r)=\frac{\omega_{\mathrm{e}}}{2\left(B_{\mathrm{e}} D_{\mathrm{e}}\right)^{1 / 2}}\left(\frac{r-r_{\mathrm{e}}}{r_{\mathrm{e}}}\right)
$$

and $b$ and $c$ are parameters defined by

$$
\begin{gathered}
b=2-\frac{1}{c}\left(\frac{7}{12}-\frac{D_{\mathrm{e}} A_{2}}{A_{0}}\right) \\
c=1+A_{1}\left(\frac{D_{\mathrm{e}}}{A_{0}}\right)^{1 / 2}
\end{gathered}
$$

TABLE 2: Spectroscopic Parameters for $\mathrm{HCl}^{53}$ All Quantities in au

$$
\begin{array}{ll}
D_{0}=1.6293169 \times 10^{-1} & \omega_{\mathrm{e}}=1.3627859 \times 10^{-2} \\
B_{\mathrm{e}}=4.826752 \times 10^{-5} & \omega_{\mathrm{e}} x_{\mathrm{e}}=2.4066110 \times 10^{-4} \\
\alpha_{\mathrm{e}}=1.3996304 \times 10^{-} & \omega_{\mathrm{e}} y_{\mathrm{e}}=1.0223128 \times 10^{-6} \\
r_{\mathrm{e}}=2.40855517 & \omega_{\mathrm{e}} z_{\mathrm{e}}=-5.54966 \times 10^{-8} \\
\mu=1785.6866 &
\end{array}
$$

TABLE 3: Numerical Coefficients for the ArO HFACE Potential: ${ }^{54}$ All Quantities in au

$\begin{array}{ll}a=111.806996 & A_{6}=4.6547873 \\ b=1.956897 & A_{8}=3.8038890 \\ r_{0}=6.4651 a_{0} & A_{10}=3.2525512 \\ C_{6}=32.17 & B_{6}=9.6802180 \\ C_{8}=589.99661 & B_{8}=7.9933059 \\ C_{10}=13176.4259 & B_{10}=6.6003615 \\ \mu=20824.7929 & \end{array}$

The values of $A_{0}, A_{1}$, and $A_{2}$ depend on experimental constants through the relations:

$$
\begin{gathered}
A_{0}=\frac{\omega_{\mathrm{e}}^{2}}{4 B_{\mathrm{e}}} \\
A_{1}=-1-\frac{\alpha_{\mathrm{e}} \omega_{\mathrm{e}}}{6 B_{\mathrm{e}}^{2}} \\
A_{2}=\frac{5}{4} A_{1}^{2}-\frac{2 \omega_{\mathrm{e}} x_{\mathrm{e}}}{3 B_{\mathrm{e}}}
\end{gathered}
$$

where $r_{\mathrm{e}}$ has the meaning assigned above, and $D_{\mathrm{e}}$ has been obtained as

$$
D_{\mathrm{e}} \approx D_{0}+\frac{1}{2} \omega_{\mathrm{e}}-\frac{1}{4} \omega_{\mathrm{e}} x_{\mathrm{e}}+\frac{1}{8} \omega_{\mathrm{e}} y_{\mathrm{e}}+\frac{1}{16} \omega_{\mathrm{e}} z_{\mathrm{e}}
$$

In turn, $D_{0}$ is the spectroscopic dissociation energy, $B_{\mathrm{e}}$ the rotational constant, $\alpha_{\mathrm{e}}$ the vibrational-rotational coupling constant, and $\omega_{\mathrm{e}}, \omega_{\mathrm{e}} x_{\mathrm{e}}, \omega_{\mathrm{e}} y_{\mathrm{e}}, \omega_{\mathrm{e}} z_{\mathrm{e}}$ are the anharmonicity constants in the Dunham series expansion. Such experimental parameters are tabulated in ref 53, and gathered in Table 2. One obtains $D_{\mathrm{e}}=0.16969 E_{\mathrm{h}}$.

To represent ArO, we have utilized the Hartree-Fock approximate correlation energy (HFACE) model $^{54}$ proposed by Varandas and Silva for diatomic interactions of both chemically stable and van der Waals molecules. It assumes the form

$$
V(r)=a \exp (-b r)-\sum_{n=6,8,10} C_{n} \frac{\chi_{n}(r)}{r^{n}}
$$

where

$$
\chi_{n}(r)=\left[1-\exp \left(-A_{n} x-B_{n} x^{2}\right)\right]^{n}
$$

and

$$
x=\frac{r}{5.5+1.25 r_{0}}
$$

The values of the relevant parameters are given in Table 3, while $D_{\mathrm{e}}=2.7979937 \times 10^{-4} E_{\mathrm{h}}$.

2.3. Eigenvalue Calculations. For comparison with the methods based on the classical framework, we evaluate also the corresponding quantum sum-over-states in eq 1. For this, we must solve the one-dimensional Schrödinger equation 


$$
\left[-\frac{\hbar^{2} \mathrm{~d}^{2}}{2 \mu \mathrm{d} r^{2}}+\frac{\hbar^{2}}{2 \mu r^{2}} l(l+1)+V(r)\right] \psi_{v l}(r)=\epsilon_{v l} \psi_{v l}(r)
$$

where $v$ and $l$ are the vibrational and rotational quantum numbers, respectively. We have employed here for its solution the well-established discrete variable representation (DVR) method $^{55-58}$ as reported by Colbert and Miller. ${ }^{57}$ For each rotational quantum number $l$, we have then used $N=500$ equally spaced DVR grid points, keeping all eigenvalues with an energy smaller than the dissociation energy $\left(\epsilon_{v l} \leq D_{\mathrm{e}}\right)$. To warrant convergence, the integration intervals were set at $(a$, $b)=\left(0.0,25.0 a_{0}\right)$ and $(a, b)=\left(3.0,50.0 a_{0}\right)$ for the for $\mathrm{HCl}$ and ArO systems, respectively. The calculations led to a total of 941 and 57 rovibrational energy levels for $\mathrm{HCl}$ and $\mathrm{ArO}$ up to $l=64$ and $l=20$, respectively. For $\mathrm{H}_{2}$ we have used the exact eigenvalues given by eq 12 , since only the vibrational partition function is calculated for this system.

\section{Corrections to the Classical Partition Function}

We now discuss briefly the methods used to apply quantum, semiclassical, and semiempirical corrections to the classical partition function. To assess their efficiency, all such methods are employed to evaluate the vibrational partition function of $\mathrm{H}_{2}$. For each method, the formulas will be written in terms of generalized coordinates $(\mathbf{q})$ and conjugate momenta (p) such that they can be applied to the rovibrational partition function of any polyatomic molecule. The calculations of the involved integrals will be carried out using the Monte Carlo technique described in section 2, with $M=10^{6}$ and $r_{\max }=25.0 a_{0}$. We will examine first the classical vibrational partition function of $\mathrm{H}_{2}$ in order to investigate the quality of the various correction schemes.

3.1. The Wigner-Kirkwood Expansion. Wigner ${ }^{14}$ and Kirkwood $^{15}$ derived a quantum mechanical version of the Boltzmann probability distribution function in phase space. Within their approach, the quantum partition function is expressed as the following expansion in powers of $\hbar^{2}$ :

$$
\begin{array}{r}
q_{\mathrm{vr}}^{\mathrm{WK}}(T)=\frac{1}{h^{n}} \iint_{\mathrm{B}} \exp \left\{-\beta H^{\mathrm{CM}}(\mathbf{q}, \mathbf{p})\right\}(1+ \\
\left.\sum_{i=1}^{\infty} \hbar^{2 i} \varphi_{i}(\mathbf{q}, \mathbf{p})\right) \mathrm{d} \mathbf{q} \mathrm{d} \mathbf{p}
\end{array}
$$

where the functions $\left\{\varphi_{i}(\mathbf{q}, \mathbf{p})\right\}$ can be obtained from a recursive formula, and $\mathrm{B}$ has the meaning previously defined. Note that eq 26 reduces to the classical expression when $\hbar \rightarrow 0$ (or $T \rightarrow$ $\infty)$. Thus, the classical partition function can be seen as the zeroth-order approximation, with the quantum effects being given by the higher-order corrections. The first term in the WK expansion assumes the form

$$
\begin{array}{r}
\varphi_{1}(\mathbf{q}, \mathbf{p})=-\frac{\beta^{2}}{8 \mu} \nabla^{2} V(\mathbf{q})+\frac{\beta^{3}}{24 \mu}\left[(\nabla V(\mathbf{q}))^{2}+\right. \\
\left.(\mathbf{p} \cdot \nabla)^{2} V(\mathbf{q}) / \mu\right]
\end{array}
$$

with terms up to sixth-order being given in ref 59. As such terms contain high-order derivatives of the potential energy surface, their application to realistic systems is difficult. For example, in the popular one-dimensional hindered-rotor case, the evaluation of terms up to $\hbar^{4}$ in the WK expansion has been carried out with the aid of a computer algebra code. ${ }^{20}$ Thus, we will limit our calculations to the term $\hbar^{2}$ in eq 26.

For diatomic molecules, eq 27 yields

$$
\begin{array}{r}
\varphi_{1}\left(r, p_{r}\right)=-\frac{\beta^{2}}{8 \mu}\left(\frac{\mathrm{d}^{2}}{\mathrm{~d} r^{2}}+\frac{1}{r} \frac{\mathrm{d}}{\mathrm{d} r}\right) V(r)+\frac{\beta^{3}}{24 \mu}\left[\left(\frac{\mathrm{d}}{\mathrm{d} r} V(r)\right)^{2}+\right. \\
\left.\frac{p_{r}^{2}}{\mu} \frac{\mathrm{d}^{2}}{\mathrm{~d} r^{2}} V(r)\right]
\end{array}
$$

and the WK vibrational partition function up to second-order assumes the form

$$
\begin{array}{r}
q_{\mathrm{v}}^{\mathrm{WK}}(T)=\frac{1}{h} \iint_{\mathrm{B}} \exp \left\{-\beta\left(\frac{p_{r}^{2}}{2 \mu}+V(r)\right)\right\}[1+ \\
\left.\hbar^{2} \varphi_{1}\left(r, p_{r}\right)\right] \mathrm{d} r \mathrm{~d} p_{r}
\end{array}
$$

with B standing for $0 \leq\left(p_{r}^{2} / 2 \mu\right)+V(r) \leq D_{\mathrm{e}}$. The results for the vibrational WK partition function of $\mathrm{H}_{2}$ obtained from eq 29 are given in Table 4. For comparison, we also give in this table the quantum and classical vibrational partition functions from eq 1 and eq 2, respectively. As it is well-known, the WK expansion up to second-order assumes negative values at low temperatures; for $\mathrm{H}_{2}$ is found to occur at $\mathrm{T} \simeq 1250 \mathrm{~K}$, in agreement with ref 22. Clearly, Table 4 shows that the results from the WK expansion start to converge to the quantum results at a lower temperature than the classical ones. This is also seen from Figure 1, where the errors relative to the quantum result $\left[\Delta q_{\mathrm{v}}^{\mathrm{WK}}=\left(q_{\mathrm{v}}^{\mathrm{WK}}-q_{\mathrm{v}}^{\mathrm{QM}}\right) / q_{\mathrm{v}}^{\mathrm{QM}}\right]$ are plotted. For example, at $T=$ $2000 \mathrm{~K}$, the error in $q_{\mathrm{v}}^{\mathrm{WK}}$ is $\simeq 11 \%$ while that of $q_{\mathrm{v}}^{\mathrm{CM}}$ is $\simeq 45 \%$. For $\mathrm{T}=9000 \mathrm{~K}$, one observes a deviation of $0.3 \%$ in $q_{\mathrm{v}}^{\mathrm{WK}}$, and of $1.8 \%$ in $q_{\mathrm{v}}^{\mathrm{CM}}$. Moreover, the WK partition function assumes accurate values $\left(\triangle q_{\mathrm{v}}^{\mathrm{WK}} \leq 0.01\right)$ for temperatures above $T=$ $3000 \mathrm{~K}$

3.2. Green-Oppenheim-Ross Expansion. The incorporation of quantum effects in classical simulations via the $\hbar^{2}$ expansion can be done by substituting the classical Hamiltonian in eq 2 by an approximate quantum Hamiltonian written in the phase space representation formalism. This approach has been suggested by Green ${ }^{23}$ and Oppenheim and Ross, ${ }^{24}$ and is frequently employed in molecular dynamics ${ }^{25,60}$ simulations. The basic equation of this approach is ${ }^{25,60}$

$$
q_{\mathrm{vr}}^{\mathrm{GOR}}(T)=\frac{1}{h^{n}} \iint_{\mathrm{B}} \mathrm{d} \mathbf{q} \mathrm{d} \mathbf{p} \exp \left\{-\beta H^{\mathrm{QM}}(\mathbf{q}, \mathbf{p})\right\}
$$

where

$$
H^{\mathrm{QM}}(\mathbf{q}, \mathbf{p})=H^{\mathrm{CM}}(\mathbf{q}, \mathbf{p})-\frac{1}{\beta} \sum_{i=1}^{\infty} \hbar^{2 i} \varphi_{i}(\mathbf{q}, \mathbf{p})
$$

and the classical Hamiltonian and functions $\left\{\varphi_{i}\right\}$ are the same as in the WK expansion. As before, we truncate the expansion at $\hbar^{2}$ term. Thus, we use

$$
\begin{array}{r}
H^{\mathrm{Q} M}(\mathbf{q}, \mathbf{p})=H^{\mathrm{CM}}(\mathbf{q}, \mathbf{p})+\frac{\beta \hbar^{2}}{24 \mu}\left\{3 \nabla^{2} V(\mathbf{q})-\right. \\
\left.\beta\left[(\nabla V(\mathbf{q}))^{2}+\frac{(\mathbf{p} \cdot \nabla)^{2}}{\mu} V(\mathbf{q})\right]\right\}
\end{array}
$$

The $q_{\mathrm{v}}^{\mathrm{GOR}}$ results obtained for $\mathrm{H}_{2}$ from eq 30 are given in Table 4. As seen, the GOR expansion leads to ill-conditioned results for low temperatures due to the fact that the Hamiltonian can assume large negatives values. Only for temperatures around $3000 \mathrm{~K}$, the method begins to give acceptable values, and only at $T \approx 4000 \mathrm{~K}$ does $\left|\triangle q_{\mathrm{v}}^{\mathrm{GOR}}\right|$ becomes smaller than $\left|\triangle q_{\mathrm{v}}^{\mathrm{CM}}\right|$, see Figure 1. However, its error relative to the quantum partition 
TABLE 4: Vibrational Partition Function ${ }^{a}$ of $\mathbf{H}_{2}$ Calculated Using Various Approaches

\begin{tabular}{|c|c|c|c|c|c|c|c|c|}
\hline$T / \mathrm{K}$ & $q_{\mathrm{v}}^{\mathrm{CM}}$ & $q_{\mathrm{v}}^{\mathrm{WK}}$ & $q_{\mathrm{v}}^{\mathrm{GOR}}$ & $q_{\mathrm{v}}^{\mathrm{LCP}}$ & $q_{\mathrm{v}}^{\mathrm{QFH}}$ & $q_{\mathrm{v}}^{\mathrm{LCP} / \mathrm{QFH}}$ & $q_{\mathrm{v}}^{\mathrm{PG}}$ & $q_{\mathrm{v}}^{\mathrm{QM}}$ \\
\hline \multirow[t]{2}{*}{300} & $4.792(-2)^{b}$ & $-8.416(-1)$ & $c$ & $7.696(-3)$ & $1.653(-9)$ & $1.065(-6)$ & $4.619(-5)$ & $3.082(-5)$ \\
\hline & $1.8(-3)^{d}$ & $7.6(-2)$ & $c$ & $2.2(-4)$ & $1.8(-11)$ & $8.4(-8)$ & $1.8(-6)$ & \\
\hline \multirow[t]{2}{*}{500} & $7.978(-2)$ & $-4.474(-1)$ & $c$ & $2.096(-2)$ & $1.400(-4)$ & $1.059(-3)$ & $2.453(-3)$ & $1.965(-3)$ \\
\hline & $2.3(-3)$ & $3.5(-2)$ & $c$ & $4.1(-4)$ & $1.4(-6)$ & $5.1(-5)$ & $7.2(-5)$ & \\
\hline \multirow[t]{2}{*}{700} & $1.118(-1)$ & $-2.611(-1)$ & $c$ & $3.991(-2)$ & $4.266(-3)$ & $1.002(-2)$ & $1.348(-2)$ & $1.166(-2)$ \\
\hline & $2.8(-3)$ & $2.1(-2)$ & $c$ & $6.2(-4)$ & $3.9(-5)$ & $3.6(-4)$ & $3.3(-4)$ & \\
\hline \multirow[t]{2}{*}{1000} & $1.600(-1)$ & $-9.824(-2)$ & $c$ & $7.706(-2)$ & $3.209(-2)$ & $4.382(-2)$ & $4.856(-2)$ & $4.444(-2)$ \\
\hline & $3.3(-3)$ & $1.2(-2)$ & $c$ & $9.4(-4)$ & $2.7(-4)$ & $1.1(-3)$ & $1.0(-3)$ & \\
\hline \multirow[t]{2}{*}{1500} & $2.410(-1)$ & $7.125(-2)$ & $c$ & $1.545(-1)$ & $1.181(-1)$ & $1.292(-1)$ & $1.338(-1)$ & $1.277(-1)$ \\
\hline & $4.1(-3)$ & $6.7(-3)$ & $c$ & $1.5(-3)$ & $8.9(-4)$ & $2.5(-3)$ & $2.3(-3)$ & \\
\hline \multirow[t]{2}{*}{2000} & $3.227(-1)$ & $1.972(-1)$ & $c$ & $2.415(-1)$ & $2.165(-1)$ & $2.244(-1)$ & $2.283(-1)$ & $2.219(-1)$ \\
\hline & $4.7(-3)$ & $4.4(-3)$ & $c$ & $2.0(-3)$ & $1.5(-3)$ & $3.5(-3)$ & $3.3(-3)$ & \\
\hline \multirow[t]{2}{*}{3000} & $4.887(-1)$ & $4.074(-1)$ & $1.434(+2)$ & $4.221(-1)$ & $4.104(-1)$ & $4.140(-1)$ & $4.167(-1)$ & $4.116(-1)$ \\
\hline & $5.7(-3)$ & $2.4(-3)$ & 3.0 & $2.9(-3)$ & $2.6(-3)$ & $5.0(-3)$ & $4.9(-3)$ & \\
\hline \multirow[t]{2}{*}{4000} & $6.584(-1)$ & $5.993(-1)$ & $6.582(-1)$ & $6.039(-1)$ & $5.981(-1)$ & $5.995(-1)$ & $6.012(-1)$ & $5.978(-1)$ \\
\hline & $6.6(-3)$ & $1.7(-3)$ & $5.5(-3)$ & $3.7(-3)$ & $3.5(-3)$ & $6.1(-3)$ & $6.0(-3)$ & \\
\hline \multirow[t]{2}{*}{5000} & $8.326(-1)$ & $7.868(-1)$ & $8.068(-1)$ & $7.875(-1)$ & $7.846(-1)$ & $7.844(-1)$ & $7.853(-1)$ & $7.833(-1)$ \\
\hline & $7.4(-3)$ & $1.4(-3)$ & $6.5(-3)$ & $4.4(-3)$ & $4.2(-3)$ & $7.1(-3)$ & $7.0(-3)$ & \\
\hline \multirow[t]{2}{*}{6000} & 1.0122 & $9.752(-1)$ & $9.860(-1)$ & $9.767(-1)$ & $9.755(-1)$ & $9.716(-1)$ & $9.718(-1)$ & $9.709(-1)$ \\
\hline & $8.1(-3)$ & $1.3(-3)$ & $7.4(-3)$ & $5.1(-3)$ & $5.0(-3)$ & $7.8(-3)$ & $7.8(-3)$ & \\
\hline \multirow[t]{2}{*}{7000} & 1.1980 & 1.1672 & 1.1739 & 1.1782 & 1.1780 & 1.1630 & 1.1626 & 1.1627 \\
\hline & $8.7(-3)$ & $1.4(-3)$ & $8.2(-3)$ & $5.7(-3)$ & $5.6(-3)$ & $8.5(-3)$ & $8.5(-3)$ & \\
\hline \multirow[t]{2}{*}{8000} & 1.3908 & 1.3645 & 1.3689 & 1.4012 & 1.4016 & 1.3601 & 1.3591 & 1.3599 \\
\hline & $9.3(-3)$ & $1.5(-3)$ & $8.9(-3)$ & $6.4(-3)$ & $6.3(-3)$ & $9.2(-3)$ & $9.1(-3)$ & \\
\hline \multirow[t]{2}{*}{9000} & 1.5906 & 1.5676 & 1.5707 & 1.6563 & 1.6571 & 1.5634 & 1.5619 & 1.5630 \\
\hline & $9.9(-2)$ & $1.7(-2)$ & $9.5(-3)$ & $7.0(-3)$ & $6.9(-3)$ & $9.8(-3)$ & $9.7(-3)$ & \\
\hline
\end{tabular}

${ }^{a}$ See the text for nomenclature. ${ }^{b}$ Given in parantheses is the power of 10 by which the numbers should be multiplied. ${ }^{c}$ Unconverged results. ${ }^{d}$ Second entry for each temperature gives the standard Monte Carlo deviation.

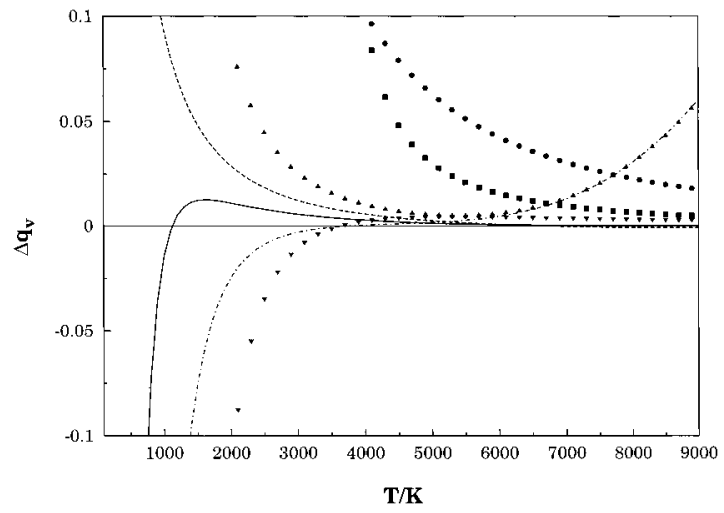

Figure 1. Relative errors of the approximate vibrational partition function of hydrogen molecule as a function of temperature: $(\bullet) \Delta$ $q_{\mathrm{v}}^{\mathrm{CM}},(\boldsymbol{\nabla}) \Delta q_{\mathrm{v}}^{\mathrm{WK}},(\boldsymbol{\square}) \Delta q_{\mathrm{v}}^{\mathrm{GOR}},(\boldsymbol{\Delta}) \Delta q_{\mathrm{v}}^{\mathrm{LCP}},(-\cdot) \Delta q_{\mathrm{v}}^{\mathrm{QFH}},(--) \Delta q_{\mathrm{v}}^{\mathrm{PG}}$, and (一) $\Delta q_{\mathrm{v}}^{\mathrm{LCP} / \mathrm{QFH}}$ (see the text for nomenclature).

function is always larger than in $q_{\mathrm{v}}^{\mathrm{WK}}$ for the temperature range considered in the present work. Convergence to $q_{\mathrm{v}}^{\mathrm{QM}}$ starts at $T$ $\geq 6900 \mathrm{~K}$.

3.3. Semiclassical Approach. The "better than classical" approach proposed by Miller ${ }^{36}$ to approximate the Boltzmann density matrix was named classical path approximation. This semiclassical approach is based upon a classical-limit approximation to the quantum mechanical time evolution propagator $U(t)$ and a relation between time and the reciprocal temperature $(t=i \hbar \beta)$. This led to a number ${ }^{36-39}$ of approximate expressions for the partition function, which differ on the way in which the potential is expanded. In particular, we employ LCP approximation given by ${ }^{36,38}$

$$
\begin{aligned}
q_{\mathrm{vr}}^{\mathrm{LCP}}(T)=\left(\frac{\mu}{2 \pi \beta \hbar^{2}}\right)^{3 n / 2} & \int \mathrm{d} \mathbf{q} \\
& \exp \left\{-\beta\left[V(\mathbf{q})+\frac{\beta^{2} \hbar^{2}}{24 \mu}(\nabla V(\mathbf{q}))^{2}\right]\right\}
\end{aligned}
$$

Note that there is no limiting condition on the energy $\beta$, with the configurational integral being solved by sampling the whole coordinate space. Note especially that to examine the vibrational partition function of a diatomic, one just requires to replace $\mathbf{q}$ by the radial coordinate.

The values of $q_{\mathrm{v}}^{\mathrm{LCP}}$ and corresponding Monte Carlo errors are given for $\mathrm{H}_{2}$ in Table 4. Moreover, the errors relative to the quantum results are displayed in Figure 1. The results show a better agreement at low temperatures when compared to the previous quantum approaches $\left(q_{\mathrm{v}}^{\mathrm{WK}}\right.$ and $\left.q_{\mathrm{v}}^{\mathrm{GOR}}\right)$. In addition $q_{\mathrm{v}}^{\mathrm{LCP}}$ does not diverge for $\mathrm{T} \rightarrow 0$. At a temperature of $1000 \mathrm{~K}$, where $q_{\mathrm{v}}^{\mathrm{WK}}$ is negative with a relative error of $321 \%$ and $q_{\mathrm{v}}^{\mathrm{GOR}}$ is very large, $q_{\mathrm{v}}^{\mathrm{LCP}}$ shows a deviation of $75 \%$. We also observe that $q_{\mathrm{v}}^{\mathrm{LCP}}$ is accurate within $10 \%$ at $T \approx 1900 \mathrm{~K}$, while converging to $q_{\mathrm{v}}^{\mathrm{QM}}$ for $T \simeq 4000 \mathrm{~K}$ (the relative errors are smaller than $1 \%$ ). However, it starts to diverge for $T>5500 \mathrm{~K}$ : at $T=$ $9000 \mathrm{~K}$ the relative error is already $\sim 6 \%$. As discussed in ref 12 , this arises due to the fact that eq 33 implicitly considers the contribution of dissociative species.

3.4. The Quadratic Feynman-Hibbs Approximation. The Feynman path integral formalism is one of the most appealing approaches to evaluate the quantum mechanical partition function. ${ }^{61}$ However, its application to large systems is computationally very demanding. ${ }^{62}$ In the context of quantum corrections, a simple approximation which was proposed initially by Feynman and $\mathrm{Hibbs}^{27}$ consists of replacing the classical potential by an effective one. In this case, the partition function assumes the form of $q^{\mathrm{CM}}$ where the integration over momenta is carried out explicitly (as in the LCP approach) and the classical potential is replaced by an effective quantum one. The simplest is the quadratic Feynman-Hibbs ${ }^{27}$ potential obtained by keeping only quadratic fluctuations around the classical paths. The QFH partition function assumes the form

$$
\begin{aligned}
q_{\mathrm{vr}}^{\mathrm{QFH}}(T)=\left(\frac{\mu}{2 \pi \beta \hbar^{2}}\right)^{3 n / 2} & \int \mathrm{d} \mathbf{q} \\
& \exp \left\{-\beta\left[V(\mathbf{q})+\frac{\beta \hbar^{2}}{24 \mu} \nabla^{2} V(\mathbf{q})\right]\right\}
\end{aligned}
$$


which has been extensively used in molecular dynamics simulations of liquids. ${ }^{18,33}$

The values of $q_{\mathrm{v}}^{\mathrm{QFH}}$ and $\Delta q_{\mathrm{v}}^{\mathrm{QFH}}$ for $\mathrm{H}_{2}$ are shown in Table 4 and Figure 1, respectively. As shown, the behavior of QFH vibrational partition function is similar to the LCP one. The basic difference occurs at low temperatures, where $q_{\mathrm{v}}^{\mathrm{QFH}}$ underestimates the exact value of the vibrational partition function while $q_{\mathrm{v}}^{\mathrm{LCP}}$ overestimates it. For example, at $T=700$ $\mathrm{K}, q_{\mathrm{v}}^{\mathrm{QFH}}$ is $63 \%$ smaller than the quantum one, whereas $q_{\mathrm{v}}^{\mathrm{LCP}}$ is larger than $q_{\mathrm{v}}^{\mathrm{QM}}$ by about $242 \%$. In general, the QFH approximation leads to results better than the LCP approximation, reaching an accuracy of $10 \%$ at $T \approx 1400 \mathrm{~K}$ and converging to the quantum sum-over-states for $T \approx 2400 \mathrm{~K}$. However, as for $q_{\mathrm{v}}^{\mathrm{LCP}}, q_{\mathrm{v}}^{\mathrm{QFH}}$ diverges for high temperatures.

3.5. Pitzer-Gwinn Approximation. The scheme developed by Pitzer and Gwinn ${ }^{41}$ has a semiempirical nature and was originally developed to include quantum corrections in the calculation of thermodynamic functions for molecules with internal rotation. In their approach, the partition function is approximated by its classical component scaled by the ratio of the quantum over classical partition functions for a reference system where both are known exactly. If the reference system is described by a harmonic potential, then one obtains ${ }^{41-43}$

$$
q_{v}^{\mathrm{PG}}(T)=q_{v}^{\mathrm{CM}}(T) \frac{q_{\mathrm{HO}}^{\mathrm{QM}}(T)}{q_{\mathrm{HO}}^{\mathrm{CM}}(T)}
$$

where $q_{\mathrm{v}}^{\mathrm{CM}}(T)$ is the classical partition function, and

$$
q_{\mathrm{HO}}^{\mathrm{QM}}(T)=\frac{\exp \left(-\beta \epsilon_{0}\right)}{1-\exp (-\beta h v)} \quad \text { and } \quad q_{\mathrm{HO}}^{\mathrm{CM}}(T)=\frac{1}{\beta h v}
$$

are, respectively, the quantum and classical partition functions for the harmonic oscillator calculated with the zero of energy at the bottom of the potential curve; $v$ is the vibrational fundamental frequency. The zero point energy $\epsilon_{0}$ appearing in the expression for $q_{\mathrm{HO}}^{\mathrm{QM}}$ can be calculated by using different methods, ${ }^{42}$ such as the harmonic and Dunham formulas. In this work, we have used the relation $\epsilon_{0}=h v / 2$. Furthermore, since the zeroth and the first vibrational levels are known, we have considered the frequency $v$ in eq 36 to be the frequency associated to the $1 \leftarrow 0$ transition. Despite its simple form, the PG correction is known to be fairly accurate and hence is frequently used. ${ }^{63}$

Numerical values of $q_{\mathrm{v}}^{\mathrm{PG}}$ and the associated Monte Carlo errors are reported for $\mathrm{H}_{2}$ in Table 4 , while $\Delta q_{\mathrm{v}}^{\mathrm{PG}}$ is depicted in Figure 1. For temperatures below $1900 \mathrm{~K}$, they show a better agreement with the quantum result than all previous corrections $\left(q_{\mathrm{v}}^{\mathrm{WK}}, q_{\mathrm{v}}^{\mathrm{GOR}}, q_{\mathrm{v}}^{\mathrm{LCP}}\right.$, and $\left.q_{\mathrm{v}}^{\mathrm{QFH}}\right)$. In fact, for temperatures of a few hundred kelvin, $q_{\mathrm{v}}^{\mathrm{PG}}$ still gives values of the same order of magnitude of the quantum ones $\left(\Delta q_{\mathrm{v}}^{\mathrm{PG}} \approx 0.50\right.$ at $T=300 \mathrm{~K}$, whereas $q_{\mathrm{v}}^{\mathrm{PG}}$ reaches an accuracy of $10 \%$ at $T \approx 900 \mathrm{~K}$ ). For intermediate temperatures, the other approaches correct better the classical partition function: the QFH partition function is better than the PG one for $1900 \leq T / \mathrm{K} \leq 5200$, and the WK expansion gives better results for $2900 \leq T / \mathrm{K} \leq 4500$. The Pitzer-Gwinn values converge to $q_{\mathrm{v}}^{\mathrm{QM}}$ for lower temperatures values than in the GOR and LCP ones: $T \approx 3200 \mathrm{~K}$ for $q_{\mathrm{v}}^{\mathrm{PG}}, T$ $\approx 6900 \mathrm{~K}$ for $q_{\mathrm{v}}^{\mathrm{GOR}}$, and $T \approx 4000 \mathrm{~K}$ for $q_{\mathrm{v}}^{\mathrm{LCP}}$. Moreover, for the highest temperature considered in the present work $(T=$ $9000 \mathrm{~K}$ ) a deviation of only $0.07 \%$ is still obtained for $q_{\mathrm{v}}^{\mathrm{PG}}$.
TABLE 5: Rovibrational Partition Function ${ }^{a}$ of $\mathrm{HCl}$ Calculated Using Various Approaches

\begin{tabular}{lllll}
\hline$T / \mathrm{K}$ & \multicolumn{1}{c}{$q_{\mathrm{vr}}^{\mathrm{CM}}$} & \multicolumn{1}{c}{$q_{\mathrm{vr}}^{\mathrm{LCP} / \mathrm{QFH}}$} & \multicolumn{1}{c}{$q_{\mathrm{vr}}^{\mathrm{QM}}$} & \multicolumn{1}{c}{$q_{\mathrm{vr}}^{\mathrm{FP} b}$} \\
\hline 300 & 1.1690 & $3.7718(-3)^{c}$ & $1.6526(-2)$ & $1.67(-2)$ \\
& $1.7(-1)^{d}$ & $7.2(-4)$ & & $8.0(-4)$ \\
400 & 2.2370 & $7.0418(-2)$ & $1.3008(-1)$ & $1.39(-1)$ \\
& $2.7(-1)$ & $1.2(-2)$ & & $5(-3)$ \\
500 & 3.6074 & $3.4172(-1)$ & $4.7205(-1)$ & $4.9(-1)$ \\
& $3.8(-2)$ & $4.7(-2)$ & & $2.0(-2)$ \\
600 & 5.2885 & $9.5499(-1)$ & 1.1538 & 1.17 \\
& $4.7(-1)$ & $1.1(-1)$ & & $2.0(-2)$ \\
900 & 12.248 & 5.4206 & 5.7147 & 5.76 \\
& $7.6(-1)$ & $3.8(-1)$ & & $7.0(-2)$ \\
1000 & 15.217 & 7.8115 & 8.0999 & \\
& $8.4(-1)$ & $4.8(-1)$ & & \\
1200 & 22.139 & 13.851 & 14.115 & 14.2 \\
& 1.0 & $6.9(-1)$ & & $2.0(-1)$ \\
2000 & 63.324 & 53.240 & 53.569 & 53.6 \\
& 1.7 & 1.5 & & $5.0(-1)$ \\
4000 & 268.19 & 257.04 & 257.62 & 257.0 \\
& 3.5 & 3.4 & & 2.0 \\
5000 & 431.70 & 420.43 & 421.11 & 420.0 \\
& 4.4 & 4.3 & & 3.0 \\
6000 & 641.27 & 629.99 & 630.74 & 636.0 \\
& 5.3 & 5.3 & & 5.0 \\
7000 & 900.13 & 888.91 & 889.65 & \\
& 6.2 & 6.2 & & \\
9000 & 1567.8 & 1557.0 & 1557.3 & \\
& 8.1 & 8.0 & & \\
& & & &
\end{tabular}

${ }^{a}$ See the text for nomenclature. ${ }^{b}$ Fourier path integral (FPI) results from ref $64 .{ }^{c}$ Given in parantheses is the power of 10 by which the numbers should be multiplied. ${ }^{d}$ Second entry for each temperature gives the standard Monte Carlo deviation.

\section{A Hybrid LCP/QFH Correction}

We have found that $q_{\mathrm{v}}^{\mathrm{WK}}$ and $q_{\mathrm{v}}^{\mathrm{GOR}}$ diverge at low temperatures because the corresponding expansions (probability distribution for WK and quantum Hamiltonian for GOR) in phase space formalism are truncated at $\hbar^{2}$. However, they both converge to the quantum sum-over-states quicker than the classical result. On the other hand, the LCP and QFH methods give close results to $q_{\mathrm{v}}^{\mathrm{QM}}$ at low temperatures: $q_{\mathrm{v}}^{\mathrm{LCP}}$ overestimates whereas $q_{\mathrm{v}}^{\mathrm{QFH}}$ underestimates the quantum vibrational partition function. Unfortunately, both the methods diverge at high temperatures since they are based on the solution of the configurational integral rather than the total phase space integral in eq 2 . In this section, we suggest an hybrid scheme which aims to keep the advantages of the LCP and QFH methods while discarding their nondesirable features.

From the GOR approach, it is easy to demonstrate ${ }^{25}$ by integrating the phase space integral in eq 32 over the momenta between $-\infty$ and $\infty$ that the effective quantum potential is given by

$$
V^{\mathrm{QM}}(\mathbf{q})=V(\mathbf{q})+\frac{\hbar^{2}}{24 \mu}\left\{2 \beta \nabla^{2} V(\mathbf{q})-\beta^{2}(\nabla \cdot V(\mathbf{q}))^{2}\right\}
$$

where the $\hbar^{2}$ term can be seen as a mixture of the correction terms in eqs 33 and 34. Moreover, we have seen in section 3.2 that the GOR approach diverges at low temperatures, which is mainly due to the fact that $-\beta^{2}(\nabla V(\mathbf{q}))^{2}$ assumes very large negative values. Conversely, the LCP method has been shown to reach large positive deviations at low temperatures. This suggests that an average of the LCP and QFH quantum effective potentials may lead to acceptable results at such lowtemperature regimes. Equivalently, the terms $\beta \nabla^{2} V(\mathbf{q})$ and $\beta^{2}(\nabla V(\mathbf{q}))^{2}$ may be thought as being of the same order of magnitude, and hence be profitably combined. Thus, our 
TABLE 6: Rovibrational Partition Function ${ }^{a}$ of ArO Calculated Using Various Approaches

\begin{tabular}{clll}
\hline$T / \mathrm{K}$ & \multicolumn{1}{c}{$q_{v r}^{\mathrm{CM}}$} & \multicolumn{1}{c}{$q_{v r}^{\mathrm{LCP} / \mathrm{QFH}}$} & \multicolumn{1}{c}{$q_{v r}^{\mathrm{QM}}$} \\
\hline 2.5 & $8.0560(-1)^{b}$ & $1.6874(-4)$ & $2.6069(-3)$ \\
& $3.8(-2)^{c}$ & $1.6(-5)$ & \\
5.0 & 3.2648 & $3.0101(-1)$ & $4.0479(-1)$ \\
& $7.8(-2)$ & $9.7(-3)$ & \\
10.0 & 13.956 & 7.6740 & 7.4753 \\
& $1.6(-1)$ & $9.1(-2)$ & \\
15.0 & 33.432 & 25.973 & 25.254 \\
& $2.3(-1)$ & $1.8(-1)$ & \\
25.0 & 93.008 & 85.760 & 83.976 \\
& $3.7(-1)$ & $3.4(-1)$ & \\
50.0 & 256.73 & 252.65 & 249.04 \\
& $6.7(-1)$ & $6.6(-1)$ & \\
75.0 & 377.50 & 375.15 & 371.48 \\
& $8.7(-1)$ & $8.7(-1)$ & \\
100.0 & 461.48 & 459.99 & 456.78 \\
& 1.0 & 1.0 &
\end{tabular}

${ }^{a}$ See the text for nomenclature. ${ }^{b}$ Given in parantheses is the power of 10 by which the numbers should be multiplied. ${ }^{c}$ Second entry for each temperature gives the standard Monte Carlo deviation.

approach consists of adding the following effective potential to the classical Hamiltonian:

$$
\begin{array}{r}
q_{v r}^{\mathrm{LCP} / \mathrm{QFH}}(T)=\frac{1}{h^{n}} \iint_{\beta} \mathrm{d} \mathbf{q d} \operatorname{p} \exp \left\{-\beta\left[H^{\mathrm{CM}}(\mathbf{q}, \mathbf{p})+\right.\right. \\
\left.\left.V^{\mathrm{eff}}(\mathbf{q})\right]\right\}
\end{array}
$$

where

$$
V^{\mathrm{eff}}(\mathbf{q})=\frac{\hbar^{2} \beta}{48 \mu}\left\{\nabla^{2} V(\mathbf{q})+\beta(\nabla V(\mathbf{q}))^{2}\right\}
$$

and $\beta$ implies the above-discussed restriction on the integration hypervolume.

For the diatomic case, the effective potential reduces to

$$
V^{\mathrm{eff}}(r)=\frac{\hbar^{2} \beta}{48 \mu}\left\{\left(\frac{\mathrm{d}^{2}}{\mathrm{~d} r^{2}}+\frac{1}{r} \frac{\mathrm{d}}{\mathrm{d} r}\right) V(r)+\beta\left(\frac{\mathrm{d}}{\mathrm{d} r} V(r)\right)^{2}\right\}
$$

\begin{tabular}{|c|c|c|c|c|c|c|c|c|c|}
\hline \multirow[b]{2}{*}{$\tau$} & \multicolumn{3}{|c|}{$\mathrm{H}_{2}$} & \multicolumn{3}{|c|}{$\mathrm{HCl}$} & \multicolumn{3}{|c|}{$\mathrm{ArO}$} \\
\hline & $T / \mathrm{K}$ & $q_{\mathrm{v}}^{\mathrm{CM}} / q_{\mathrm{v}}^{\mathrm{QM}}$ & $q_{\mathrm{v}}^{\mathrm{LCP} / \mathrm{QFH}} / q_{\mathrm{v}}^{\mathrm{QM}}$ & $T / \mathrm{K}$ & $q_{\mathrm{vr}}^{\mathrm{CM}} / q_{\mathrm{vr}}^{\mathrm{QM}}$ & $q_{\mathrm{vr}}^{\mathrm{LCP} / \mathrm{QFH}} / q_{\mathrm{vr}}^{\mathrm{QM}}$ & $T / \mathrm{K}$ & $q_{\mathrm{vr}}^{\mathrm{CM}} / q_{\mathrm{vr}}^{\mathrm{QM}}$ & $q_{\mathrm{vr}}^{\mathrm{LCP} / \mathrm{QFH}} / q_{\mathrm{vr}}^{\mathrm{QM}}$ \\
\hline 0.2 & 623.2 & 14.763 & 0.7738 & 427.0 & 13.255 & 0.6012 & 4.36 & 13.266 & 0.6114 \\
\hline 0.3 & 934.8 & 4.1841 & 0.9733 & 640.4 & 3.9282 & 0.8557 & 6.54 & 3.8321 & 0.9257 \\
\hline 0.4 & 1246.5 & 2.4141 & 1.0075 & 853.9 & 2.3090 & 0.9386 & 8.72 & 2.2414 & 1.0125 \\
\hline 0.5 & 1558.1 & 1.8100 & 1.0126 & 1067.4 & 1.7500 & 0.9718 & 10.90 & 1.6977 & 1.0302 \\
\hline 0.6 & 1869.7 & 1.5291 & 1.0118 & 1280.9 & 1.4889 & 0.9848 & 13.08 & 1.4471 & 1.0307 \\
\hline 0.7 & 2181.3 & 1.3742 & 1.0100 & 1494.3 & 1.3450 & 0.9899 & 15.26 & 1.3114 & 1.0282 \\
\hline 0.8 & 2492.9 & 1.2791 & 1.0081 & 1707.8 & 1.2568 & 0.9922 & 17.44 & 1.2302 & 1.0257 \\
\hline 0.9 & 2804.6 & 1.2164 & 1.0066 & 1921.3 & 1.1986 & 0.9935 & 19.61 & 1.1779 & 1.0239 \\
\hline 1.0 & 3116.2 & 1.1726 & 1.0053 & 2134.8 & 1.1582 & 0.9944 & 21.79 & 1.1423 & 1.0226 \\
\hline 1.2 & 3739.4 & 1.1171 & 1.0035 & 2561.7 & 1.1070 & 0.9957 & 26.15 & 1.0984 & 1.0209 \\
\hline 1.4 & 4362.6 & 1.0844 & 1.0023 & 2988.7 & 1.0769 & 0.9966 & 30.51 & 1.0732 & 1.0196 \\
\hline 1.6 & 4985.9 & 1.0634 & 1.0015 & 3415.7 & 1.0578 & 0.9972 & 34.87 & 1.0573 & 1.0184 \\
\hline 1.8 & 5609.1 & 1.0492 & 1.0009 & 3842.6 & 1.0448 & 0.9976 & 39.23 & 1.0465 & 1.0172 \\
\hline 2.0 & 6232.4 & 1.0392 & 1.0005 & 4269.6 & 1.0356 & 0.9980 & 43.59 & 1.0388 & 1.0161 \\
\hline 2.5 & 7790.4 & 1.0241 & 1.0002 & 5337.0 & 1.0217 & 0.9985 & 54.49 & 1.0269 & 1.0135 \\
\hline
\end{tabular}

From eq 38, one then obtains the rovibrational partition function as

TABLE 7: Ratio of Various Partition Functions ${ }^{a}$ as a Function of the Reduced Temperature $\tau$ for $\mathrm{H}_{2}, \mathrm{HCl}$, and $\mathrm{ArO}$

${ }^{a}$ See the text for nomenclature.

$$
\begin{aligned}
& q_{v r}^{\mathrm{LCP} / \mathrm{QFH}}(T)=\frac{1}{h^{3}} \iint_{\beta} \mathrm{d} r \mathrm{~d} \theta \mathrm{d} \phi \mathrm{d} p_{r} \mathrm{~d} p_{\theta} \mathrm{d} p_{\phi} \\
& \quad \exp \left\{-\beta\left[\frac{p_{r}^{2}}{2 \mu}+\frac{p_{\theta}^{2}}{2 \mu r^{2}}+\frac{p_{\phi}^{2}}{2 \mu r^{2} \sin ^{2} \theta}+V(r)+V^{\text {eff }}(r)\right]\right\}
\end{aligned}
$$

while the vibrational partition function assumes the form

$$
\begin{aligned}
q_{v}^{\mathrm{LCP} / \mathrm{QFH}}(T)=\frac{1}{h} \iint_{\beta} \mathrm{d} r \mathrm{~d} p_{r} \\
\quad \exp \left\{-\beta\left[\frac{p_{r}^{2}}{2 \mu}+V(r)+V^{\mathrm{eff}}(r)\right]\right\}
\end{aligned}
$$

The results of such an hybrid approach to the vibrational partition function are presented in Table 4 for the vibrational partition function of $\mathrm{H}_{2}$. Clearly, for low and medium temperatures, the agreement of $q_{\mathrm{v}}^{\mathrm{LCP} / \mathrm{QFH}}$ with the quantum results is good. Moreover, no sign of divergence is manifest at high temperatures which contrasts with the LCP and QFH approaches. In fact, $q_{\mathrm{v}}^{\mathrm{LCP} / \mathrm{QFH}}$ reaches an accuracy of $10 \%$ at $T \simeq$ $750 \mathrm{~K}$ while starting to converge to $q_{\mathrm{v}}^{\mathrm{QM}}$ at $T \simeq 2100 \mathrm{~K}$. In addition, at $T=9000 \mathrm{~K}$, a deviation of only $0.03 \%$ is obtained. Although $q_{\mathrm{v}}^{\mathrm{LCP} / \mathrm{QFH}}$ gives the best overall results when compared with the quantum ones, the $q_{\mathrm{v}}^{\mathrm{PG}}$ and $q_{\mathrm{v}}^{\mathrm{QFH}}$ methods are still the best for $T \leq 700 \mathrm{~K}$ and $2600 \leq T / \mathrm{K} \leq 4800$, respectively. These features can also be seen from Figure 1.

To test further the validity of the LCP/QFH approach suggested in the present work, we have computed the rovibrational partition function of $\mathrm{HCl}$ and $\mathrm{ArO}$ by using eq 41. The results are given in Table 5 and Table 6. For comparison, we also give the exact quantum and classical results from eq 1 and eq 2. For comparison, we also report the results obtained by using the Fourier path integral formalism ${ }^{64}\left(q_{\mathrm{vr}}^{\mathrm{FPI}}\right)$ based on the same potential curve.

Table 5 shows that $q_{\mathrm{vr}}^{\mathrm{LCP} / \mathrm{QFH}}$ is much more accurate than $q_{\mathrm{vr}}^{\mathrm{CM}}$ over the whole range of temperatures. In comparison with $q_{\mathrm{vr}}^{\mathrm{QM}}$ and $q_{\mathrm{vr}}^{\mathrm{FPI}}$, the agreement is seen to be satisfactory even at low temperatures. Specifically, at $T=600 \mathrm{~K}$, where $q_{\mathrm{vr}}^{\mathrm{CM}}$ has an error of $358 \%$ and the Fourier path integral formalism has an error of $1.4 \%$, one finds for $q_{\mathrm{vr}}^{\mathrm{LCP} / \mathrm{QFH}}$ a deviation of $17 \%$. At medium temperatures $(\mathrm{T} \approx 2000 \mathrm{~K}) q_{\mathrm{vr}}^{\mathrm{LCP} / \mathrm{QFH}}$ is comparable in accuracy to $q_{\mathrm{vr}}^{\mathrm{FPI}}$, while becaming more accurate with increasing temperature.

As expected, ${ }^{13}$ Table 6 shows that similar considerations apply for $\mathrm{ArO}$ but for much lower temperatures than in the cases of 
$\mathrm{H}_{2}$ and $\mathrm{HCl}$. For example, at $T=5.0 \mathrm{~K}$ where $q_{\mathrm{vr}}^{\mathrm{CM}}$ shows a deviation of $700 \%, q_{\mathrm{vr}}^{\mathrm{LCP} / \mathrm{QFH}}$ has an error of only $25.6 \%$. Such an observation can be rationalized from the zero point of energy $\left(\epsilon_{0}\right)$ values of the three molecules: $\epsilon_{0}=2165.866 \mathrm{~cm}^{-1}$ for $\mathrm{H}_{2}, \epsilon_{0}=1483.759 \mathrm{~cm}^{-1}$ for $\mathrm{HCl}$, and $\epsilon_{0}=15.148 \mathrm{~cm}^{-1}$ for ArO. For this, we use the following reduced temperature ${ }^{13}$

$$
\tau=\frac{k_{\mathrm{B}} T}{\epsilon_{0}}=0.695 \frac{T}{\epsilon_{0}}
$$

where $T$ is in $K$, and $\epsilon_{0}$ in $\mathrm{cm}^{-1}$. Table 7 reports the values of the ratios $q_{\mathrm{vr}}^{\mathrm{CM}} / q_{\mathrm{vr}}^{\mathrm{QM}}$ and $q_{\mathrm{vr}}^{\mathrm{LCP} / \mathrm{QFH}} / q_{\mathrm{vr}}^{\mathrm{QM}}$ as a function of $\tau$; for reference, the corresponding $T$ values are also tabulated. Clearly, similar trends are observed for the deviations of $q^{\mathrm{LCP} / \mathrm{QFH}}$ from $q^{\mathrm{QM}}$ as a function of $\tau$. For example, at $\tau=0.5, q^{\mathrm{CM}}$ shows a deviation of $81 \%$ for $\mathrm{H}_{2}, 75 \%$ for $\mathrm{HCl}$, and $70 \%$ for $\mathrm{ArO}$, while the corresponding deviations for $q^{\mathrm{LCP} / \mathrm{QFH}}$ are $1.3 \%, 2.8 \%$, and $3.0 \%$. Moreover, for $\tau \simeq 1.2, q^{\mathrm{CM}}$ shows an error of $10 \%$ for the three diatomic systems while $q^{\mathrm{LCP} / \mathrm{QFH}}$ reaches the same accuracy at $\tau \simeq 0.25$ for $\mathrm{H}_{2}$ and $\mathrm{ArO}$, and at $\tau \simeq 0.35$ for $\mathrm{HCl}$. Thus, the LCP/QFH hybrid method proposed here can reliably be used to calculate the rovibrational partition functions of diatomic molecules for temperatures above $T=0.3 \epsilon_{0} / k_{\mathrm{B}}$.

\section{Conclusions}

Several quantum, semiclassical, and semiempirical corrections to the classical partition function of molecular systems have been analized. In addition, a novel hybrid procedure (LCP/QFH) has been proposed. A numerical application to $\mathrm{H}_{2}$ has shown that this new method performs generally better than previous ones, namely for temperatures above $\sim 700 \mathrm{~K}$. Similar performances are anticipated for the $\mathrm{HCl}$ and $\mathrm{ArO}$ cases also reported in this work. In fact, it blends the advantages of two popular semiclassical methods (LCP and QFH) but restricted to the bound phase space. Although applications were done only for diatomic molecules, there is no reason of principle why it should not work equally well for larger polyatomics. Work along these lines is currently in progress.

Acknowledgment. This work has the support of Fundação para a Ciência e Tecnologia, Portugal, under program PRAXIS XXI. It has also benefited from an EC grant under Contract CHRX-CT 94-0436. One of us (F.V.P.) also acknowledges partial financial support from Fundação Coordenação de Aperfeiçoamento de Pessoal de Nível Superior (CAPES, Brazil).

\section{References and Notes}

(1) McQuarrie, D. A. Statistical Mechanics; Harper and Row: New York, 1976.

(2) Johnston, H. S. Gas-Phase Reaction Rate Theory; Ronald: New York, 1966.

(3) Truhlar, D. G.; Isaacson, A. D.; Garrett, B. G. In Theory of Chemical Reaction; Baer, M., Ed.; CRC: Boca Raton, 1985; Part IV, p 65.

(4) Bačíc, Z.; Light, J. C. Annu. Rev. Phys. Chem. 1989, 40, 469.

(5) Tennyson, J. In Theoretical High-Resolution Molecular Spectroscopy; Jensen, P., Bunker, P. R., Eds.; Wiley: New York, 2000.

(6) Prudente, F. V.; Costa, L. S.; Acioli, P. H. J. Phys. B: At. Mol. Opt. Phys. 2000, 33, R285.

(7) Neale, L.; Tennyson, J. Astrophys. J. 1995, 454, L169.

(8) Harris, G. J.; Viti, S.; Mussa, H. Y.; Tennyson, J. J. Chem. Phys. 1998, 109, 7197.

(9) Partridge, H.; Schenke, D. W. J. Chem. Phys. 1997, 106, 4618 6325

10) Koput, J.; Carter, S.; Handy, N. C. J. Phys. Chem. A 1998, 102,

(11) Landau, L.; Lifshitz, E. Statistical Physics; Pergamon Press: New York, 1969

(12) Riganelli, A.; Prudente, F. V.; Varandas, A. J. C. J. Phys. Chem. A. Submitted for publication.

(13) Riganelli, A.; Prudente, F. V.; Varandas, A. J. C. Phys. Chem Chem. Phys. 2000, 2, 4121.

(14) Wigner, E. Phys. Rev. 1932, 40, 749

(15) Kirkwood, J. G. Phys. Rev. 1933, 44, 31.

(16) Powles, J. G.; Rickayzen, G. Mol. Phys. 1979, 38, 1875.

(17) Gibson, W. G. Mol. Phys. 1975, 1, 1.

(18) Sesé, L. M. Mol. Phys. 1993, 78, 1167.

(19) Thirumalai, D.; Hall, R. W.; Berne, B. J. J. Chem. Phys. 1984 $81,2523$.

(20) Witschel, W.; Hartwigsen, C. Chem. Phys. Lett. 1997, 273, 304

(21) Taubmann, G. J. Phys. B: At. Mol. Opt. Phys. 1995, 28, 533.

(22) Taubmann, G.; Witschel, W.; Schoendorff, L. J. Phys. B: At. Mol. Opt. Phys. 1999, 32, 2859

(23) Green, H. S. J. Chem. Phys. 1951, 19, 955

(24) Oppenheim, I.; Ross, J. Phys. Rev. 1957, 107, 28

(25) Allen, M. P.; Tildesley, D. J. Computer Simulations of Liquids; Clarendron Press: Oxford, 1987.

(26) Feynman, R. P. Rev. Mod. Phys. 1948, 20, 367.

(27) Feynman, R. P.; Hibbs, A. R. Quantum Mechanics and Statistical Mechanics; McGraw-Hill: New York, 1965.

(28) Doll, J. D.; Myers, L. E. J. Chem. Phys. 1979, 71, 2880

(29) Giacchetti, R.; Tognetti, V. Phys. Rev. Lett. 1985, 55, 912

(30) Giacchetti, R.; Tognetti, V. Phys. Rev. B 1986, 33, 7647.

(31) Feynman, R. P.; Kleinert, H. Phys. Rev. A 1986, 34, 5080.

(32) Janke, W.; Kleinert, H. Chem. Phys. Lett. 1987, 137, 162.

(33) Sesé, L. M. Mol. Phys. 1993, 78, 1167.

(34) Sesé, L. M. Mol. Phys. 1994, 81, 1297.

(35) Messina, M.; Schenter, G. K.; Garrett, C. B. J. Chem. Phys. 1993, 98,4120 .

(36) Miller, W. H. J. Chem. Phys. 1971, 55, 3146.

(37) Hornstein, S. M.; Miller, W. H. Chem. Phys. Lett. 1972, 13, 298

(38) Miller, W. H. J. Chem. Phys. 1973, 58, 1664.

(39) Stratt, R. M.; Miller, W. H. J. Chem. Phys. 1977, 67, 5894.

(40) Fukui, K.; Cline, J. I.; Frederick, J. H. J. Chem. Phys. 1997, 107, 4551

(41) Pitzer, K. S.; Gwinn, W. D. J. Chem. Phys. 1942, 10, 428.

(42) Isaacson, A. D.; Truhlar, D. G. J. Chem. Phys. 1981, 75, 4090

(43) Isaacson, A. D.; Truhlar, D. G. J. Chem. Phys. 1984, 80, 2888

(44) Frankiss, S. G. J. Chem. Soc., Faraday Trans. 1974, 70, 1516.

(45) Hui-Yun, P. J. Chem. Phys. 1987, 87, 4846.

(46) Riganelli, A.; Wang, W.; Varandas, A. J. C. J. Phys. Chem. A 1999, 103,8303 .

(47) Barker, J. R. J. Phys. Chem. 1987, 91, 3849

(48) Varandas, A. J. C.; Silva, J. D. J. Chem. Soc., Faraday Trans. 2 1992, 88,941

(49) Peña-Gallego, A.; Abreu, P. E.; Varandas, A. J. C. J. Phys. Chem A 2000, 104, 6241.

(50) Morse, P. M. Phys. Rev. 1929, 34, 57.

(51) Pauling, L.; Wilson, E. B. Introduction to Quantum Mechanics; McGraw-Hill: New York, 1935.

(52) Hulbert, H. M.; Hirschfelder, J. O. J. Chem. Phys. 1941, 9, 61

(53) Huber, K. P.; Herzberg, G. Molecular Spectra and Molecular Structure IV Constants of Diatomic Molecules; Van Nostrand Reinhold: New York, 1979

(54) Varandas, A. J. C.; Silva, J. D. J. Chem. Soc., Faraday Trans. 2 1986, 82,593 .

(55) Baye, D.; Heenen, P. H. J. Phys. A: Math. Gen. 1986, 19, 2041

(56) Muckerman, J. T. Chem. Phys. Lett. 1990, 173, 200.

(57) Colbert, D. T.; Miller, W. H. J. Chem. Phys. 1992, 96, 1982

(58) Prudente, F. V.; Costa, L. S.; Soares Neto, J. J. J. Mol. Struct. (THEOCHEM) 1997, 394, 169.

(59) Haberlandt, R. Z. Phys. Chem. 1974, 255, 1136.

(60) Singer, J. V. L.; Singer, K. CCP5 Quarterly 1984, 14, 24

(61) Topper, R. Q. Adv. Chem. Phys. 1999, 105, 117.

(62) Srinivasan, J.; Volobuev, Y. L.; Mielke, S. L.; Truhlar, D. G. Comput. Phys. Commun. 2000, 128, 446.

(63) McClurg, R. B.; Flagan, R. C.; Goddard, W. A., III J. Chem. Phys 1997, 106, 6675 .

(64) Topper, R. Q.; Towa, G. J.; Truhlar, D. G. J. Chem. Phys. 1992 97,3668 .

(65) Marston, C. C.; Balint-Kurti, G. G. J. Chem. Phys. 1989, 91, 3571 